Alma Mater Studiorum - Università di Bologna DEPARTMENT OF ECONOMICS

\title{
Substitution Effects in Intertemporal Problems
}

\section{Davide Dragone}

Paolo Vanin

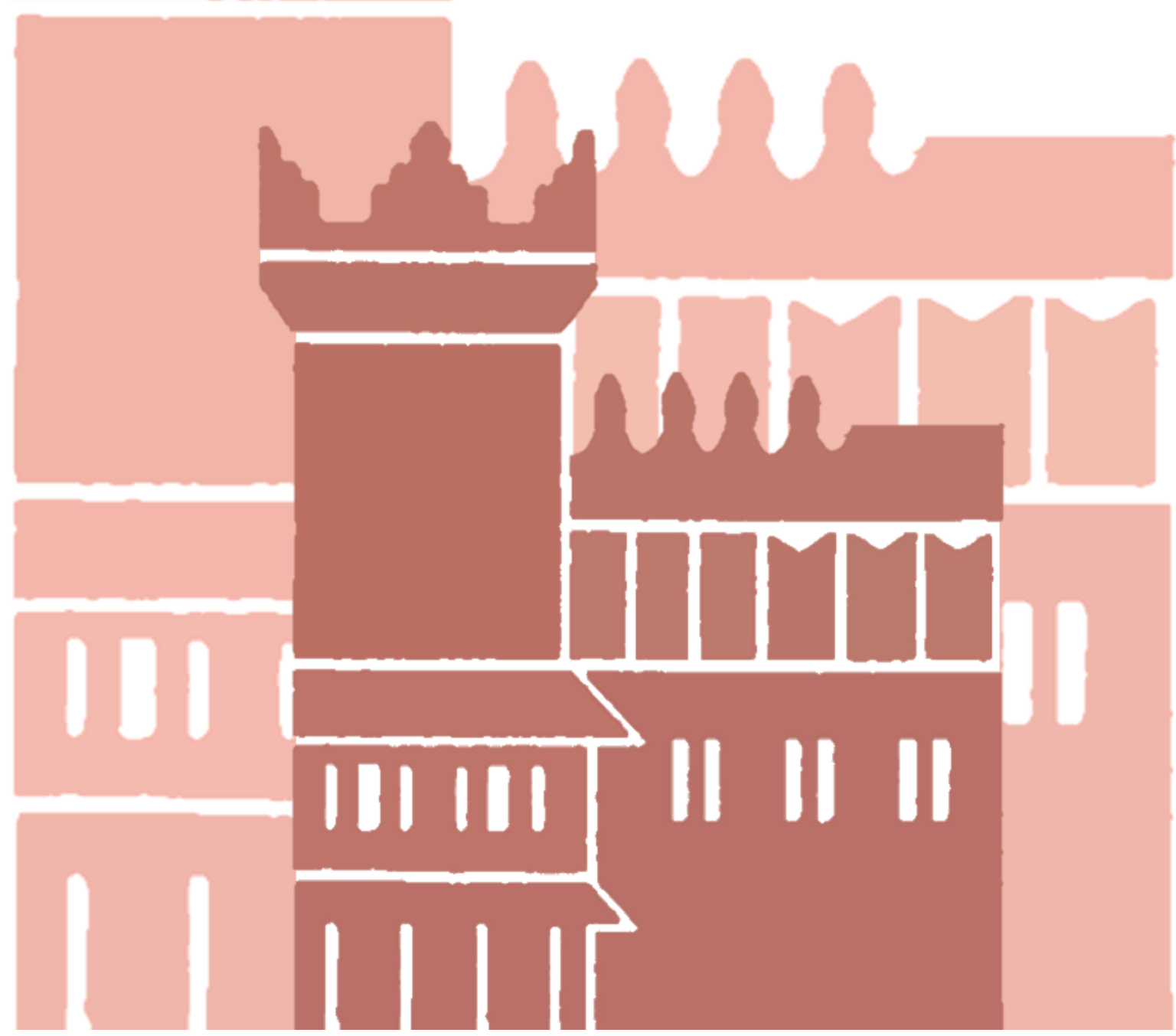




\title{
Substitution Effects in Intertemporal Problems*
}

\author{
Davide Dragone \\ Paolo Vanin \\ University of Bologna ${ }^{\dagger} \quad$ University of Bologna
}

May 5, 2020

\begin{abstract}
We consider a broad class of intertemporal economic problems and we characterize the short and long-run response of the demand for a good to a permanent increase in its market price. Depending on the interplay between self-productivity and time discounting, we show that dynamic substitution effects can generate price elasticities of opposite sign in the short and in the long run.
\end{abstract}

JEL Classification: D11, D91, I00

Keywords: Intertemporal substitution, Price elasticity, Tax reforms

${ }^{*}$ We thank for useful comments and suggestions Antonio Cabrales, Giacomo Calzolari, Chris Cronin, Vincenzo Denicolò, Matthew Ellman, James Heckman, Paolo Manasse, Antonio Minniti, Tito Pietra, Holger Strulik, Giulio Zanella and the participants to the 2015 ASSET Conference in Granada, the 2015 EEA Congress in Mannheim, the 2015 UPF-GPEFM Alumni Meeting in Barcelona, the 2016 PET Conference in Rio de Janeiro, the 2016 SIE Conference in Milan, the 2016 Brucchi Luchino Workshop in Bologna, the 2017 Workshop on Stochastic Optimal Control in Gotheborg, the 2017 Health and Labor Conference in Essen, the Department of Applied Economics in Palma, and the Department of Economics in Bologna. The usual disclaimer applies.

${ }^{\dagger}$ University of Bologna, Department of Economics, Piazza Scaravilli 2, 40126, Bologna, Italy; phone: +39-0512098880; e-mail: davide.dragone@unibo.it

${ }^{\ddagger}$ University of Bologna, Department of Economics, Piazza Scaravilli 2, 40126, Bologna, Italy; phone: +39-0512098120; e-mail: paolo.vanin@unibo.it 


\section{Substitution Effects in Intertemporal Problems}

by Davide Dragone, and Paolo Vanin

\section{NON-TECHNICAL SUMMARY}

The law of demand is a basic concept in economics: when the price of a good increases, one typically expects a reduction in the quantity demanded. This basic insight from static consumer theory does not necessarily extend to an intertemporal framework, in which current choices produce effects that persist over time. Think, for instance, of labor supply choices that affect human capital accumulation, or medical care affecting individual health.

In this paper we show that the law of demand fails when the effects of current choices are amplified over time. In this case, after a price increase, the quantity demanded increases at some point in time. Consider, as an example, the accumulation of health deficits, which typically happens at a faster rate, the higher the number of existing health deficits. If medical care becomes more expensive and one reacts by reducing medical care today, health deficits will accumulate, and this will happen at faster and faster rate over time. Ultimately, to avoid excessive health deficits, medical care will need to progressively increase until it is higher than it would have been without the price increase. In this case after a price increase the quantity demanded falls in the short run and increases in the long run. We show that this outcome occurs when a consumer is relatively impatient. The opposite case, which corresponds to an increase in medical care in the short run, and a subsequent reduction in the long run, occurs if she is sufficiently patient.

The case presented above features self-productivity, which occurs when there is a positive feedback that amplifies current behavior. When instead the effects of current actions get dampened over time, a case called self-depletion, we show that price increases induce lower levels of consumption at any point in time. In this case, the static intuition of the law of demand extends better to dynamic consumer behavior. Notably, however, consumption needs not decrease progressively over time. On the contrary, there can be an overreaction in the short run (i.e., a large consumption drop), which is then followed by a partial recovery in the long run. To highlight the contrast between static and dynamic consumer theory in the sharpest way, we characterize dynamic reactions to a price increase abstracting from income effects, that is in a context in which the law of demand necessarily holds in a static framework. 


\section{Introduction}

Price effects and intertemporal choices are at the heart of economic analysis. In static consumer problems with two goods, it is well known that substitution effects are always negative, so that consumers respond to an increase in the relative price of a good by reducing its consumption. In dynamic extensions of such problems, in which present choices affect future conditions through some relevant state variable, such as habit, human capital or health, consumers' response may be substantially different. While this has long been informally known, perhaps surprisingly no general characterization is available.

In this paper, we characterize substitution effects for a broad class of intertemporal consumer and worker problems. We identify time discounting and self-productivity as key determinants. A state variable is self-productive or self-depleting, depending on whether it contributes positively or negatively to its own accumulation. Under self-productivity we show that dynamic substitution effects have opposite sign in the short and in the long run, with the discount rate determining whether consumption initially falls and eventually increases, or the other way around. Under self-depletion, instead, short and long-run responses have the same negative sign.

As en example, think of an agent that chooses between schooling and working over time. If human capital is self-productive (i.e., if the net production of new "knowledge" increases in the agent's stock of existing "knowledge"), we show that a wage increase induces patient agents to work less and study more in the short run, in order to work more and reap the benefits of the larger stock of human capital in the long run; impatient agents respond in the opposite way. If human capital is instead self-depleting (for instance due to depreciation), short and long-run responses have the same sign: after a wage increase agents work more both in the short and in the long run and invest less in human capital.

Our results contribute to different strands of literature. First, we characterize dynamic substitution effects in a general setup. This sheds light on the key assumptions on selfproductivity and discounting that produce differences and commonalities among the apparently widely different models of health behavior, human capital, endogenous preferences, addiction and habit formation that originate from Grossman, 1972; Blinder and Weiss, 1976; Stigler and Becker, 1977; Becker and Murphy, 1988; Carroll et al., 2000.

Second, our paper contributes to the literature on the comparative statics and comparative dynamics properties of intertemporal optimization models (see, among others, Oniki, 
1973, Epstein, 1978, Otani, 1982, and Caputo, 1990, 1997). We show under which conditions dynamic substitution effects are positive or negative. An empirical implication is that heterogeneity in individual discount rates can produce an attenuation bias in estimated price responses. This result is particularly relevant when writing and solving computational models that estimate the dynamic responses to tax changes or income subsidies (see Hall, 2010, and Ljungqvist and Sargent, 2012, for an overview). An implication for policy making is that there are conditions under which policies that seem effective in the short run backfire in the long run, and vice versa.

Third, our paper relates to the literature on taxation in models with endogenous human capital, which has devoted substantial attention to the role of asymmetric information, agedependent taxation and stochastic returns, but less to the aspects that are the focus of the present paper. ${ }^{1}$ We take a complementary perspective: we abstract from all those elements to show in the cleanest way how the short and long-run response to labor income taxes depend on the interplay between self-productivity and time discounting. More broadly, we contribute to the large literature on taxation in dynamic models investigating, e.g., health behavior, addiction or obesity (see Cawley and Ruhm, 2012, for an overview).

The paper is structured as follows. Section 2 presents the general intertemporal problem and the main results. Sections 3 and 4 specialize the results to consumer and labor supply problems. Section 5 concludes.

\section{An intertemporal consumer/worker problem}

Consider the following intertemporal model, which nests consumer and labor supply problems as special cases:

$$
\begin{array}{ll}
\max _{x, y} & \int_{0}^{\infty} e^{-\rho t} U(x(t), y(t), Z(t)) \mathrm{d} t \\
\text { s.t. } & \dot{Z}(t)=f(x(t), Z(t)) \\
& \dot{A}(t)=r A(t)+M(t)+g(x(t), Z(t), p)-y(t) \\
& A(0)=A^{0}, \quad Z(0)=Z^{0}
\end{array}
$$

\footnotetext{
${ }^{1}$ See Makris and Pavan (2018) for a discussion, and in particular, among others, the works of Kapička (2006, 2015); Krause (2009); Best and Kleven (2013); Stantcheva (2017); Kapička and Neira (2019).
} 
where $x \geq 0$ and $y \geq 0$ are consumption goods, $Z$ is any form of "capital" (say, human capital, habit, health or addiction), and $A$ represents assets. ${ }^{2}$ Equation (2) describes the law of motion of state variable $Z$, and equation (3) describes the dynamic budget constraint. Parameter $\rho>0$ is the intertemporal discount rate. To maintain generality, we make no functional form assumptions on utility or the law of motion of $Z .^{3}$

Based on equation (2), we introduce the following classification (subscripts denote partial derivatives):

Definition 1 State variable $Z$ is self-depleting if $f_{Z}<0$, and self-productive if $f_{Z}>0$.

The case of self-depleting $Z$ covers all intertemporal problems where the state variable contributes to its dynamics only through depreciation. This is the case, for instance, of Grossman (1972)'s health accumulation model, Becker and Murphy (1988)'s model of rational addiction, and the models of habits formation considered in Carroll et al. (2000) and Chetty and Szeidl (2016), which share the common assumption $\dot{Z}=\mathfrak{f}(x)-\delta Z$ with $\mathfrak{f}, \mathfrak{f}^{\prime}>0$. The case of self-productive $Z$ includes the models of human capital considered, among others, in Weiss (1972) and Cunha and Heckman (2007), or Dalgaard and Strulik (2014)'s model of health deficit accumulation, in which the state variable contributes to its own accumulation according to $\dot{Z}=\mathfrak{f}(Z)-x$. In richer models where, e.g., $\dot{Z}=\mathfrak{f}(x, Z)-\delta Z$, the state variable can be self-depleting under certain conditions, and self-productive in others, as shown in the labor supply models treated in Section 4.

Equation (3) represents the dynamics of asset accumulation, where $r$ is the interest rate, $M \geq 0$ is an exogenous instantaneous income flow and $y$ is the numeraire good. The formulation of (3) is flexible enough to encompass consumption as well as labor supply choices. In consumer problems, $g(x, Z, p)=m(Z)-p x$, where $m(Z)$ is the endogenous component of income (if any) and $p x$ is expenditure on $x$ at the relative price $p$. Hence in a consumer problem the budget constraint equation becomes

$$
\dot{A}=r A+M+m(Z)-p x-y
$$

In labor supply problems, $g(x, Z, p)=p L(x, Z)$, and the budget constraint is

$$
\dot{A}=r A+M+p L(x, Z)-y
$$

\footnotetext{
${ }^{2}$ Henceforth the time arguments are omitted to simplify notation.

${ }^{3}$ The case where the evolution of $Z$ also depends on $y$, i.e., $\dot{Z}=f(x, y, Z)$ is studied in the Appendix.
} 
where $x$ represents leisure, $L(x, Z)$ is effective labor (which may depend on $Z$, e.g. if $Z$ is human capital), $L_{x}<0$, and $p$ is the market wage of effective labor.

Problem (1)-(4) is solved applying Pontryagin's maximum principle, as detailed in the Appendix. We assume concavity of the corresponding Hamiltonian function, that the market interest rate $r$ is equal to the discount rate $\rho$, and that price changes are compensated to keep the marginal utility of assets constant (for a similar approach see, e.g., Heckman, 1974; Becker and Murphy, 1988). This form of compensation is convenient because it neutralizes income effects, so that dynamic price effects are entirely driven by dynamic substitution effects. ${ }^{4}$ The corresponding system of optimal trajectories for $x$ and $y$ is

$$
\begin{aligned}
\dot{x} & =\frac{\mathcal{H}_{y y}}{\mathcal{H}_{x y}^{2}-\mathcal{H}_{x x} \mathcal{H}_{y y}}\left[\left(f_{Z}-\rho\right)\left(U_{x}+g_{x} U_{y}\right)-f_{x}\left(U_{Z}+g_{Z} U_{y}\right)\right] \\
\dot{y} & =-\frac{\mathcal{H}_{x y}}{\mathcal{H}_{y y}} \dot{x}
\end{aligned}
$$

where $\mathcal{H}$ represents the Hamiltonian function associated to the maximization problem.

Equating to zero expressions (2), (3), (7), and (8) yields the steady state $\left(x^{L}, y^{L}, Z^{L}, A^{L}\right)$. Our goal is to establish how an unexpected permanent change in price affects consumption when the system is at a steady state with saddle-point stability. For expositional simplicity, in the proceeding we focus on $x$ and $Z$, and we report the corresponding analysis for $y$ and $A$ in the Appendix.

We investigate dynamic substitution effects over two different time-horizons. The shortrun effect describes the consumption response on impact, when the price change is announced and implemented. The short-run effect is computed considering the policy function $\hat{x}(Z, A)$ leading to the steady state and computing $x_{p}^{S} \equiv \partial \hat{x}\left(Z^{L}, A^{L}\right) / \partial p .^{5}$ The long-run effect describes the change in the steady state values $x_{p}^{L} \equiv \partial x^{L} / \partial p$ and $Z_{p}^{L} \equiv \partial Z^{L} / \partial p$.

Proposition 1 Consider an unexpected permanent change in price p, occurring when all variables are at their saddle-point-stable steady state level, and compensated to maintain the

\footnotetext{
${ }^{4}$ The main insights generalize to the case in which the interest rate depends on the level of assets, as it is common in macroeconomic models, and price changes are not compensated to maintain the marginal utility of wealth constant (see Dragone and Vanin, 2015).

${ }^{5}$ Under specific assumptions one can write the closed-form expression of $\hat{x}(Z, A)$ as, e.g., in Becker and Murphy (1988)'s rational addiction model. For our results, however, this is not needed.
} 
marginal utility of wealth constant. The short and long-run responses of $x$ and $Z$ are

$$
\begin{aligned}
x_{p}^{S} & =a\left[f_{x} g_{p Z}-\left(f_{Z}-\rho\right) g_{x p}\right] \varepsilon \\
x_{p}^{L} & =a\left[f_{x} g_{p Z}-\left(f_{Z}-\rho\right) g_{x p}\right] f_{Z} \\
Z_{p}^{L} & =-a\left[f_{x} g_{p Z}-\left(f_{Z}-\rho\right) g_{x p}\right] f_{x}
\end{aligned}
$$

where concavity and saddle-point stability imply $a, \varepsilon, f_{x} / f_{Z}<0$.

Term $\varepsilon$ is the negative eigenvalue associated to the Jacobian matrix computed at the steady state, whereas $a$ is a function of the primitives of the model (see equations 65 and 74 in the Appendix). Given that the expression in square brackets is the same in all equations, the relation between the short and long-run price response is particularly simple:

Proposition 2 The short and long-run price responses are related as follows:

$$
\begin{aligned}
x_{p}^{L} & =\frac{f_{Z}}{\varepsilon} x_{p}^{S} \\
Z_{p}^{L} & =-\frac{f_{x}}{f_{Z}} x_{p}^{L} .
\end{aligned}
$$

Hence:

- If $Z$ is self-depleting:

$-x_{p}^{S}$ and $x_{p}^{L}$ have the same sign

- Demand for $x$ is more elastic in the short run than in the long run if $f_{Z}>\varepsilon$, and more rigid otherwise,

- If $Z$ is self-productive, $x_{p}^{S}$ and $x_{p}^{L}$ have opposite sign,

- $Z_{p}^{L}$ has the same sign as $x_{p}^{L}$.

These results are general and they highlight the importance of self-productivity and selfdepletion for the sign and magnitude of price responses over time. The following sections, focusing on consumer and labor supply problems, show how the interplay between selfproductivity and time discounting determines the specific sign of the short and long-run price response. 


\section{Consumer problem}

In a consumer problem, the dynamic budget constraint is (5) because $g_{p Z}=0$ and $g_{x p}=-1$. Hence the price responses (9)-(11) of Proposition 1 simplify as follows

$$
\begin{aligned}
x_{p}^{S} & =a\left(f_{Z}-\rho\right) \varepsilon \\
x_{p}^{L} & =a\left(f_{Z}-\rho\right) f_{Z} \\
Z_{p}^{L} & =-a\left(f_{Z}-\rho\right) f_{x}
\end{aligned}
$$

This allows being more specific about the direction of the price responses:

Proposition 3 In a dynamic consumer problem:

1. If $Z$ is self-depleting, price responses of $x$ are always negative and the state variable $Z$ decreases $\left(x_{p}^{S}, x_{p}^{L}, Z_{p}^{L}<0\right)$

2. If $Z$ is self-productive, the sign of price responses depends on $\rho$ relative to $f_{Z}$. Specifically:

- If the consumer is sufficiently impatient $\left(\rho>f_{Z}\right)$, consumption of $x$ decreases on impact and increases in the long-run $\left(x_{p}^{S}<0, x_{p}^{L}>0\right)$; the state variable $Z$ increases $\left(Z_{p}^{L}>0\right)$.

- If the consumer is sufficiently patient $\left(\rho<f_{Z}\right)$, consumption of $x$ increases on impact and decreases in the long-run $\left(x_{p}^{S}>0, x_{p}^{L}<0\right)$; the state variable $Z$ decreases $\left(Z_{p}^{L}<0\right)$.

The above Proposition shows how the specific sign of the short and long-run price response depends on the interplay between $f_{Z}$ and $\rho$. When $Z$ is self-depleting, a forwardlooking agent substitutes consumption away from the more expensive good and, relative to the initial steady state, she reduces the quantity demanded at any point in time, as shown in Figure $1 .^{6}$ This is similar to what happens in a static model.

A common example of this case are consumer problems in which the law of motion is of the following kind

$$
\dot{Z}(t)=x^{\alpha}-\delta Z
$$

\footnotetext{
${ }^{6}$ Whether there is overshooting in the short relative to the long run depends on whether the policy function is upward or, as in Figure 1, downward sloping.
} 


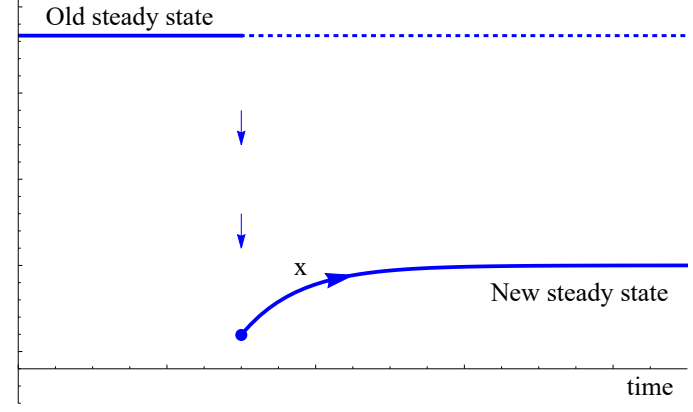

Time path of $x$

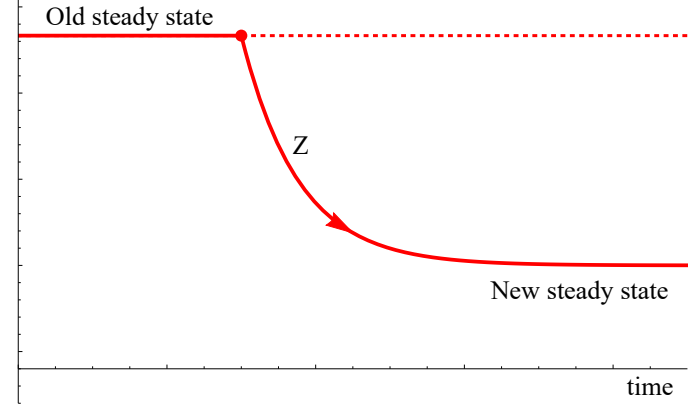

Time path of $Z$

Figure 1: Self-depleting $\left(f_{Z}<0\right)$ : Short and long-run response to a price increase. Consumption of $x$ decreases both on impact and in the long run, with overshooting in the short run if $\varepsilon<f_{Z}$. Left panel: time path for $x$. Right panel: time path for $Z$.

with $\alpha \in(0,1]$ and $\delta \in(0,1)$. The interpretation of $x$ and $Z$ depends on the specific application. In habit models and in rational addiction models, $x$ is consumption and $Z$ is the addiction stock or the habit stock (Becker and Murphy, 1988; Chetty and Szeidl, 2016). In health accumulation models, $x$ is health investment, and $Z$ is the health condition (Grossman, 1972; Galama and Kapteyn, 2011). While the role of $x$ and $Z$ in the utility function depends on the specific model, these differences are irrelevant for our results. ${ }^{7}$ In fact, inspection of the dynamics of $Z$ immediately reveals that $Z$ is self-depleting because $f_{Z}=-\delta<0$. Hence using Proposition 3 we can immediately predict that price effects are negative, both in the short and in the long run.

If instead $Z$ is self-productive, whether the short-run response is positive or negative (remembering that the long-run response has opposite sign), depends on the time discount rate $\rho$. The intuition behind this result lies in the tension between self-productivity and impatience, because self-productivity amplifies over time the consequences of current behavior, while time discounting reduces the relevance of such future consequences. If impatience dominates $\left(\rho>f_{Z}\right)$, present outcomes matter relatively more than future ones and the agent immediately reduces consumption of good $x$ when it becomes more expensive. The

\footnotetext{
${ }^{7}$ For example, in addiction and taste formation models, consumption capital raises the marginal utility of $x\left(U_{x Z}>0\right)$ : the more I listen to music the more I appreciate it, the more I consume drugs, the more I crave for them. In habit formation models, instead, $U_{x Z}<0$ : the more I get used to a good, the less I value it. See Dragone and Ziebarth (2017) for an analysis of the two cases in the context of consumption of novel vs familiar goods.
} 


$$
\rho>f_{Z}
$$

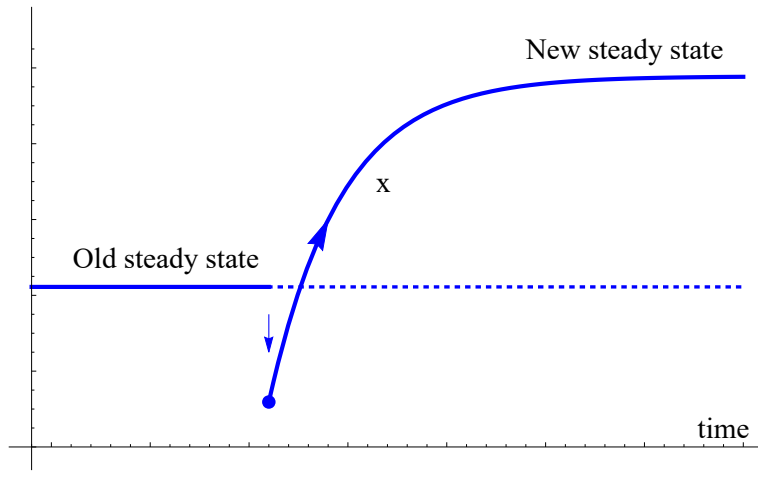

Time path of $x$

$$
\rho<f_{Z}
$$

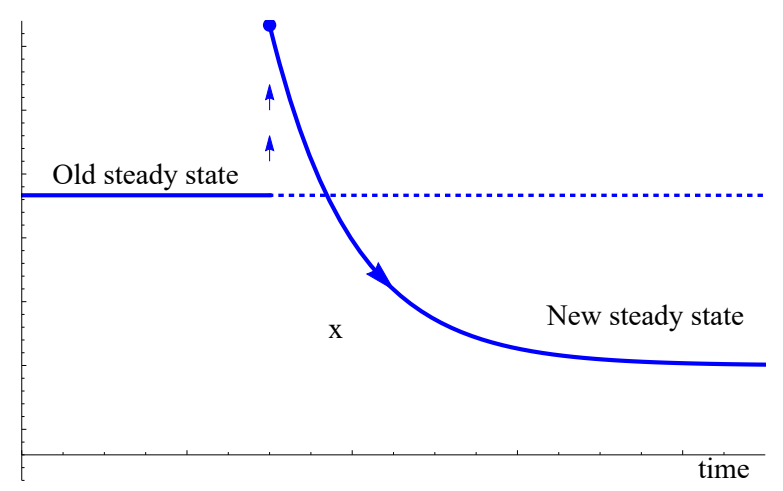

Time path of $x$

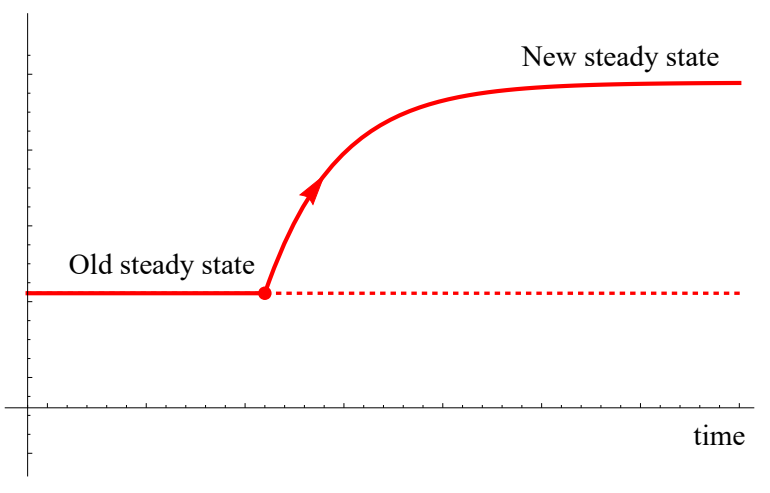

Time path of $Z$

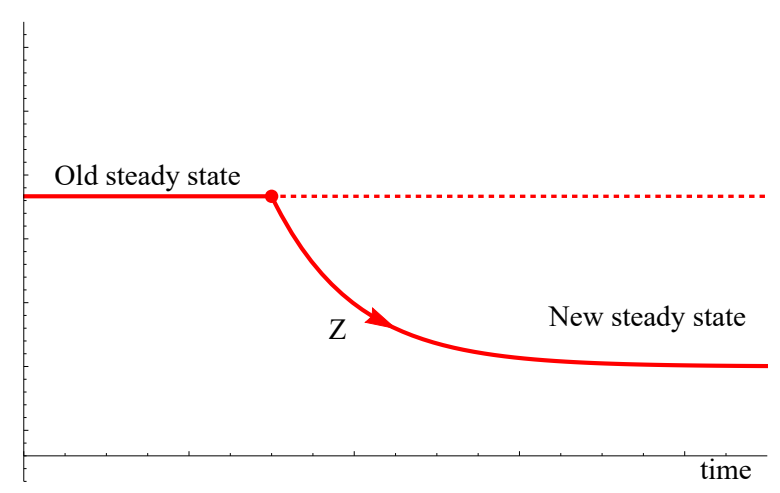

Time path of $Z$

Figure 2: Self-productive $\left(f_{Z}>0\right)$ : Short and long-run responses of $x$ to a price increase have opposite sign. After a price increase, a relatively impatient agent $\left(\rho>f_{Z}\right)$ reduces consumption on impact (as in a static model), and increases it in the long run. A relatively patient agent $\left(\rho<f_{Z}\right)$ does the opposite: she increases consumption in the short run and decreases it (as in a static model) in the long run. Left panel: time path for $x$. Right panel: time path for $Z$. 
emphasis on short-run outcomes, however, produces a persistent increase in the state variable that will eventually lead the agent to reverse her initial choice and, in the long run, increase consumption of $x$ (Figure 2, upper panel). ${ }^{8}$ If instead the agent is sufficiently patient $\left(f_{Z}>\rho\right)$, in the long run she aims at consuming less of the relatively more expensive good (as in a static model). In order to do so, she is willing to bear the short-run cost of higher consumption, which persistently decreases $Z$ and, over time, allows for lower consumption (Figure 2, lower panel).

As an example of self-productivity in consumer problems, consider the case in which law of motion is

$$
\dot{Z}=\delta Z-x^{\alpha}
$$

For instance, this is the case of the Dalgaard and Strulik (2014) and Dragone and Strulik (2017)'s model of health deficit accumulation, where $Z$ is the stock of health deficits and $x$ is health care investment. Since $Z$ is self-productive $\left(f_{Z}=\delta>0\right)$, health care has opposite short and long-run price effects (Proposition 2). Accordingly, if the agent is sufficiently patient, after an increase in the price of health care she will demand more health care in the short run and less in the long run. If instead she is sufficiently impatient, the opposite holds (Proposition 3).

\section{Labor supply and human capital accumulation}

Results are slightly more complex, but qualitatively similar for labor supply problems, in which budget constraint (6) applies. The price responses (9)-(11) of Proposition 1 are

$$
\begin{aligned}
x_{p}^{S} & =a\left[f_{x} L_{Z}-\left(f_{Z}-\rho\right) L_{x}\right] \varepsilon \\
x_{p}^{L} & =a\left[f_{x} L_{Z}-\left(f_{Z}-\rho\right) L_{x}\right] f_{Z} \\
Z_{p}^{L} & =-a\left[f_{x} L_{Z}-\left(f_{Z}-\rho\right) L_{x}\right] f_{x}
\end{aligned}
$$

Comparing these expressions to the analog expressions (14)-(16) of consumer problems, the only difference is the content in square brackets. Accordingly, one can state the exact

\footnotetext{
${ }^{8}$ With self-productivity it must be the case that, in steady state, $f_{x}<0$. Hence a short-run reduction in consumption triggers an increase in the state variable, followed by further subsequent increases due to self-productivity. Since the policy function is upward-sloping, consumption will track the evolution of the state variable that was originated by the initial change in consumption.
} 
equivalent of Proposition 3 (which we do not report here for brevity), with the only difference that here the critical value of $\rho$ is $f_{Z}-f_{x} L_{Z} / L_{x}$, rather than just $f_{Z}$.

Further insights can be obtained considering an example where, unlike equations (17) and (18), the sign of $f_{Z}$ in steady state depends on the parameters of the model. Consider a schooling model where $Z$ is human capital and $x$ is time devoted to schooling. ${ }^{9}$ The agent's problem can be written as:

$$
\begin{aligned}
\max _{\{x, y\}} & \int_{0}^{\infty} e^{-\rho t} u(x, y) \mathrm{d} t \\
\text { s.t. } & \dot{Z}=x^{\alpha} Z^{\gamma}-\delta Z \\
& \dot{A}=r A+M+p L(x, Z)-y
\end{aligned}
$$

where $\alpha, \gamma, \delta>0$, effective labor $L(x, Z)$ is increasing in human capital and decreasing in non-labor (schooling) time $\left(L_{Z}>0, L_{x}<0\right)$, and $p$ is the wage rate of effective labor.

Human capital is produced with schooling and existing human capital, and it depreciates over time. The law of motion (23) thus entails a self-productive component (the first term) and a self-depleting component (the second term), whose relative value in steady state only depends on the value of $\gamma$ : human capital $Z$ is self-productive in steady state if $\gamma>1$ (i.e., when there are increasing marginal returns to "knowledge" in the production of new "knowledge") and self-depleting if $\gamma<1$ (i.e., when marginal returns are decreasing). ${ }^{10}$

Determining which case is empirically relevant is outside the scope of this paper. ${ }^{11}$ For our purposes, it suffices to observe that, due to Proposition 2, whether $\gamma$ is larger or smaller than one is enough to predict whether in this model the short and long-run response of labor supply to a wage increase have the same sign $(\gamma<1)$ or opposite sign $(\gamma>1)$. In the

\footnotetext{
${ }^{9}$ Labor supply models fall into two broad classes: training models, which focus on the direct investment in human capital through either schooling or on-the-job training (as pioneered in Ben-Porath, 1967; Heckman, 1976; Blinder and Weiss, 1976), and learning-by-doing (or experience) models, which consider human capital accumulation as a byproduct of work activity (see Arrow, 1962; Weiss, 1972, for seminal contributions). Both classes of models have been very influential in the literature, and they are both described by problem (1)-(4) and by (6).

${ }^{10}$ To see it, observe that in steady state the requirement $\dot{Z}=0$ implies $x^{\alpha} Z^{\gamma-1}=\delta$. Since $f_{Z}=\gamma x^{\alpha} Z^{\gamma-1}-$ $\delta$, we can replace and get $f_{Z}=\delta(\gamma-1)$, which implies that $f_{Z}>0$ if and only if $\gamma>1$.

${ }^{11}$ Heckman et al. (1998) and Kapička (2006, 2015), among others, assume decreasing returns $(\gamma<1)$, but Trostel (2004) presents evidence that marginal returns are increasing $(\gamma>1)$ for low levels of human capital and decreasing $(\gamma<1)$ for high levels.
} 
latter case, under more specific functional assumptions, Proposition 3 allows determining the conditions under which labor supply first increases and then decreases, or the other way around.

\section{Discussion and conclusion}

This paper studies short and long-run price effects for a broad class of dynamic consumer/worker problems. We provide a theoretical answer to the following simple but fundamental questions. Are dynamic substitution effects always negative? Can they change sign over different time horizons? Can demand be more elastic in the short than in the long run? On what primitives of the model does it depend?

We first characterize short and long-run price responses in a general model that encompasses as special cases models of endogenous preferences, health, and human capital. We find that short and long-run substitution effects may have opposite sign. Under assumptions that are commonly used in the literature, this possibility materializes when the state variable is self-productive, a condition that can be satisfied, for instance, in models of human capital accumulation and endogenous preferences, in which "capital" is an input for its own accumulation. With self-productive capital, after a price increase impatient agents reduce consumption today and increase it tomorrow, while patient ones do the opposite. If instead the state variable is self-depleting, as it easily happens in models with capital depreciation

(including those of rational addiction and health capital), both short and long-run price responses are negative.

In most models used in the literature, our results allow for an immediate assessment of the sign of price responses over time by simple inspection of the intertemporal problem. We illustrate this possibility considering workhorse models of consumption and labor supply that have been extensively used in the literature. For example, in consumer problems with health capital (Grossman, 1972, Galama and Van Kippersluis, 2018) the law of motion features self-depletion, hence health investment is predicted to decrease both in the short and in the long run when its price increases. On the contrary, health deficit accumulation models feature self-productivity (Dalgaard and Strulik, 2014), hence the price response in the short and in the long run will have opposite sign. Models of human capital often involve a self-productive and a self-depleting element (see, e.g., Ben-Porath, 1967, Heckman, 1976, 
Blinder and Weiss, 1976 and Magnac et al., 2018). In such a case, the determination of the short and long-run substitution effect depends on which of the two effects dominates in steady state.

As intertemporal consumer problems are a building block of dynamic macroeconomic models, our results are also relevant for business cycle and growth theories, although an explicit analysis of general equilibrium models, possibly with a stochastic component, is outside the scope of the present paper. While an infinite time horizon is relevant in many applications and may represent a finite but uncertain life duration, it would be interesting to extend the analysis to a finite (and certain) time horizon, as well as to the effects of temporary price changes. This is left for future research.

\section{References}

Arrow, K. J. (1962). The economic implications of learning by doing. The Review of Economic Studies 29(3), 155-173.

Barro, R. J. and X. Sala-i Martin (2004). Economic growth. Mc-Grow-Hill, New York.

Becker, G. S. and K. M. Murphy (1988). A theory of rational addiction. The Journal of Political Economy 96(4), 675-700.

Ben-Porath, Y. (1967). The production of human capital and the life cycle of earnings. The Journal of Political Economy 75(4), 352-365.

Best, M. C. and H. J. Kleven (2013). Optimal income taxation with career effects of work effort. Mimeo, LSE.

Blinder, A. S. and Y. Weiss (1976). Human capital and labor supply: A synthesis. The Journal of Political Economy 84(3), 449-72.

Caputo, M. R. (1990). Comparative dynamics via envelope methods in variational calculus. The Review of Economic Studies 57(4), 689-697.

Caputo, M. R. (1997). The qualitative structure of a class of infinite horizon optimal control problems. Optimal Control Applications and Methods 18(3), 195-215. 
Carroll, C. D., J. Overland, and D. N. Weil (2000). Saving and growth with habit formation. The American Economic Review 90(3), 341-355.

Cawley, J. and C. J. Ruhm (2012). The economics of risky health behaviors. Handbook of Health Economics 2, 95-199.

Chetty, R. and A. Szeidl (2016). Consumption commitments and habit formation. Econometrica $84(2), 855-890$.

Cunha, F. and J. Heckman (2007). The economics of human development: The technology of skill formation. The American Economic Review 97(2), 31-47.

Dalgaard, C.-J. and H. Strulik (2014). Optimal aging and death: understanding the Preston curve. Journal of the European Economic Association 12(3), 672-701.

Dockner, E. J., S. Jorgensen, N. V. Long, and G. Sorger (2000). Differential Games in Economics and Management Science. Cambridge University Press.

Dragone, D. and H. Strulik (2017). Human health and aging over an infinite time horizon. Department of Economics Working Paper 1104, University of Bologna.

Dragone, D. and P. Vanin (2015). Price effects in the short and in the long run. Department of Economics Working Paper 1040, University of Bologna.

Dragone, D. and N. R. Ziebarth (2017). Non-separable time preferences, novelty consumption and body weight: Theory and evidence from the East German transition to capitalism. Journal of Health Economics 51, 41-65.

Epstein, L. G. (1978). The Le Châtelier principle in optimal control problems. Journal of Economic Theory 19(1), 103-122.

Galama, T. and A. Kapteyn (2011). Grossman's missing health threshold. Journal of Health Economics 30(5), 1044-1056.

Galama, T. J. and H. Van Kippersluis (2018). A theory of socio-economic disparities in health over the life cycle. The Economic Journal 129(617), 338-374.

Grossman, M. (1972). On the concept of health capital and the demand for health. The Journal of Political Economy 80(2), 223-55. 
Hall, R. E. (2010). Forward-Looking Decision Making. Princeton University Press.

Heckman, J. (1974). Life cycle consumption and labor supply: An explanation of the relationship between income and consumption over the life cycle. The American Economic Review 64(1), 188-194.

Heckman, J. J. (1976). A life-cycle model of earnings, learning, and consumption. The Journal of Political Economy 84(2), S9-S44.

Heckman, J. J., L. Lochner, and C. Taber (1998). Tax policy and human-capital formation. The American Economic Review 88(2), 293-297.

Kapička, M. (2006). Optimal income taxation with human capital accumulation and limited record keeping. Review of Economic Dynamics 9(4), 612-639.

Kapička, M. (2015). Optimal Mirrleesean taxation in a Ben-Porath economy. American Economic Journal: Macroeconomics 7(2), 219-48.

Kapička, M. and J. Neira (2019). Optimal taxation with risky human capital. American Economic Journal: Macroeconomics 11(4), 271-309.

Krause, A. (2009). Optimal nonlinear income taxation with learning-by-doing. Journal of Public Economics 93(9-10), 1098-1110.

Ljungqvist, L. and T. J. Sargent (2012). Recursive Macroeconomic Theory. MIT press.

Magnac, T., N. Pistolesi, and S. Roux (2018). Post-schooling human capital investments and the life cycle of earnings. Journal of Political Economy 126(3), 1219-1249.

Makris, M. and A. Pavan (2018). Taxation under learning-by-doing. Mimeo, Northwestern University.

Mangasarian, O. L. (1966). Sufficient conditions for the optimal control of nonlinear systems. SIAM Journal on Control 4 (1), 139-152.

Oniki, H. (1973). Comparative dynamics (sensitivity analysis) in optimal control theory. Journal of Economic Theory 6(3), 265-283.

Otani, K. (1982). Explicit formulae of comparative dynamics. International Economic Review 23(2), 411-419. 
Seierstad, A. and K. Sydsaeter (1977). Sufficient conditions in optimal control theory. International Economic Review 18(2), 367-391.

Stantcheva, S. (2017). Optimal taxation and human capital policies over the life cycle. Journal of Political Economy 125(6), 1931-1990.

Stigler, G. J. and G. S. Becker (1977). De gustibus non est disputandum. The American Economic Review 67(2), 76-90.

Trostel, P. A. (2004). Returns to scale in producing human capital from schooling. Oxford Economic Papers 56(3), 461-484.

Weiss, Y. (1972). On the optimal lifetime pattern of labour supply. The Economic Journal 82(328), 1293-1315. 


\section{A Mathematical appendix}

\section{A.1 Solving the general intertemporal problem}

In this Appendix we derive the solution for the general intertemporal problem in which the law of motion depends on both consumption goods and on the state variable $Z$, i.e.

$$
\begin{array}{ll}
\max _{x, y} & \int_{0}^{\infty} e^{-\rho t} U(x(t), y(t), Z(t)) \mathrm{d} t \\
\text { s.t. } & \dot{Z}(t)=f(x(t), y(t), Z(t)) \\
& \dot{A}(t)=r A(t)+M(t)+g(x(t), Z(t), p)-y(t) \\
& A(0)=A_{0}, \quad Z(0)=Z_{0},
\end{array}
$$

where $r=\rho>0$. The case presented in the main text just amounts to assuming that $f_{y}=0$. The associated current-value Hamiltonian function is:

$$
\mathcal{H}(x, y, Z, A, \mu, \lambda ; p, M)=U(x, y, Z)+\lambda[r A+M+g(x, Z, p)-y]+\mu f(x, y, Z)
$$

where $\lambda$ and $\mu$ are the costate variables associated to states $A$ and $Z$, respectively. The following conditions are necessary for an internal solution:

$$
\begin{aligned}
\mathcal{H}_{x} & =U_{x}(x, y, Z)+\lambda g_{x}(x, Z, p)+\mu f_{x}(x, y, Z)=0 \\
\mathcal{H}_{y} & =U_{y}(x, y, Z)-\lambda+\mu f_{y}(x, y, Z)=0 \\
\dot{\mu} & =\rho \mu-\mathcal{H}_{Z}(x, y, Z, A, \mu, \lambda ; p, M) \\
\dot{\lambda} & =\lambda(\rho-r)
\end{aligned}
$$

together with (26), (27), (28), and with the transversality conditions $\lim _{t \rightarrow \infty} e^{-\rho t} \mu(t) Z(t)=$ 0 and $\lim _{t \rightarrow \infty} e^{-\rho t} \lambda(t) A(t)=0$. The above conditions are also sufficient for a maximum if $\mathcal{H}(x, y, Z, A, \mu, \lambda ; p, M)$ is concave in state and control variables (Mangasarian, 1966; Seierstad and Sydsaeter, 1977). We assume that this is indeed the case and that $\mathcal{H}_{x x}$ and $\mathcal{H}_{y y}$ are strictly negative.

The first order conditions (30)-(31) determine the optimal value of $x$ and $y$ as functions of the state and costate variables, of the market price and of the exogenous component of income:

$$
\begin{aligned}
& x^{*}=x^{*}(Z, A, \mu, \lambda ; p, M) \\
& y^{*}=y^{*}(Z, A, \mu, \lambda ; p, M) .
\end{aligned}
$$


Replacing $\left(x^{*}, y^{*}\right)$ in (26), (27), (32) and (33) yields the optimal dynamics of state and costate variables: ${ }^{12}$

$$
\begin{aligned}
\dot{Z} & =f\left(x^{*}, y^{*}, Z\right) \\
\dot{A} & =r A+M+g\left(x^{*}, Z, p\right)-y^{*} \\
\dot{\mu} & =\rho \mu-\mathcal{H}_{Z}\left(x^{*}, y^{*}, Z, A, \mu, \lambda ; p, M\right) \\
\dot{\lambda} & =\lambda(\rho-r) .
\end{aligned}
$$

The solution $\{Z(t), A(t), \mu(t), \lambda(t)\}$ of the above system of differential equations represents the optimal trajectory of the state and costate variables, given the initial values of the state variables, the transversality conditions, $p$ and $M$ (among the other variables). This solution, once plugged in (34) and (35), determines the time-path of $x$ and $y$.

Note that, since in this model $r=\rho$, equation (39) implies that the shadow price of assets is constant over time, $\lambda(t)=\bar{\lambda}$ for all $t$. In general, $\bar{\lambda}$ depends on the parameters of the model, possibly including market prices and the exogenous component of income, i.e. $\bar{\lambda}=\bar{\lambda}(p, M)$. Its specific value is obtained by imposing additional constraints. For example, one can reasonably assume that assets go to zero (or some positive value) in steady state. With $\lambda=\bar{\lambda}$, the dimensionality of the system of differential equations effectively reduces to three equations only $(36,37$ and 38$)$. Given the initial values of the state variables and the transversality conditions, the solution $\{Z(t), A(t), \mu(t)\}$ of the reduced system of differential equations represents the optimal trajectory of the state and costate variables, which depends on $p$ and $M$, and that determines the optimal path of $x$ and $y$.

We focus on solutions leading to a saddle-point-stable steady state

$$
\begin{aligned}
x^{L} & =x^{*}\left(Z^{L}, A^{L}, \mu^{L}, \bar{\lambda} ; p, M\right) \\
y^{L} & =y^{*}\left(Z^{L}, A^{L}, \mu^{L}, \bar{\lambda} ; p, M\right) .
\end{aligned}
$$

where

$$
Z^{L}=Z^{L}(p, M) ; \quad A^{L}=A^{L}(p, M) ; \quad \mu^{L}=\mu^{L}(p, M) ;
$$

are the steady-state values of state and costate variables that satisfy (36), (37) and (38) with equality.

\footnotetext{
${ }^{12}$ Alternatively, one could derive the solution in terms of state and control variables. We opt for the current procedure to highlight the dynamics of the states and the corresponding shadow prices, and to show how our method can be applied to problems with any number of control variables.
} 
To assess the saddle-point stability of the steady state, consider the determinant of the 3-dimensional Jacobian matrix $J$ associated to (36), (37) and (38), with $\lambda=\bar{\lambda}$ :

$$
\begin{aligned}
|J|= & \rho f_{Z}\left(\rho-f_{Z}\right)+\frac{\rho}{\mathcal{H}_{x x} \mathcal{H}_{y y}-\mathcal{H}_{x y}^{2}}\left\{\left(f_{y} \mathcal{H}_{x Z}-f_{x} \mathcal{H}_{y Z}\right)^{2}-\left(f_{y}^{2} \mathcal{H}_{x x}-2 f_{x} f_{y} \mathcal{H}_{x y}+f_{x}^{2} \mathcal{H}_{y y}\right) \mathcal{H}_{Z Z}\right. \\
& \left.-\left(2 f_{Z}-\rho\right)\left[\left(f_{y} \mathcal{H}_{x y}-f_{x} \mathcal{H}_{y y}\right) \mathcal{H}_{x Z}+\left(f_{x} \mathcal{H}_{x y}-f_{y} \mathcal{H}_{x x}\right) \mathcal{H}_{y Z}\right]\right\}
\end{aligned}
$$

The steady state has saddle point stability when the Jacobian matrix $J$ admits one negative eigenvalue $\varepsilon$ (there is at most one), implying $|J|<0$.

The response of the steady state to price changes determines the long-run price response. To determine the short-run response, we consider how the saddle path leading to the steady state changes after a price shock. Notice that the saddle path can either be expressed as a function of the state variables (the feedback representation of the solution) or as a function of time (open loop solution, Dockner et al., 2000; Barro and Sala-i Martin, 2004). Here we focus on the feedback representation. As $\lambda$ is fixed, it will be enough to consider how the saddle path of $\mu$,

$$
\hat{\mu}(Z, A ; p, M)
$$

changes when $p$ or $M$ change. This information will be used to obtain the short-run response of $x$ and $y$. Plugging (44) into (34) and (35) yields the saddle paths of $x$ and $y$ in feedback form, i.e., the policy functions of $x$ and $y$ as functions of the state variables, given $p$ and $M$ :

$$
\begin{aligned}
& \hat{x}=\hat{x}(Z, A ; p, M)=x^{*}(Z, A, \hat{\mu}(Z, A ; p, M), \bar{\lambda}(p, M) ; p, M) \\
& \hat{y}=\hat{y}(Z, A ; p, M)=y^{*}(Z, A, \hat{\mu}(Z, A ; p, M), \bar{\lambda}(p, M) ; p, M) .
\end{aligned}
$$

\section{A.2 Long-run substitution effects}

Consider (40) and (41). The change of steady state consumption of $x$ after an increase in price $p$ is computed as follows:

$$
\begin{aligned}
& x_{p}^{L}=\frac{\partial x^{*}}{\partial p}+\frac{\partial x^{*}}{\partial Z} Z_{p}^{L}+\frac{\partial x^{*}}{\partial A} A_{p}^{L}+\frac{\partial x^{*}}{\partial \mu} \mu_{p}^{L}+\frac{\partial x^{*}}{\partial \lambda} \frac{\partial \bar{\lambda}}{\partial p} \\
& y_{p}^{L}=\frac{\partial y^{*}}{\partial p}+\frac{\partial y^{*}}{\partial Z} Z_{p}^{L}+\frac{\partial y^{*}}{\partial A} A_{p}^{L}+\frac{\partial y^{*}}{\partial \mu} \mu_{p}^{L}+\frac{\partial y^{*}}{\partial \lambda} \frac{\partial \bar{\lambda}}{\partial p}
\end{aligned}
$$

where $\partial \bar{\lambda} / \partial p=0$ if the price change is compensated so as to leave the marginal value of assets $\bar{\lambda}$ constant. 
To compute the above equations, apply Cramer's rule to the first order conditions (30) and (31). This yields:

$$
\begin{aligned}
\frac{\partial x^{*}}{\partial p} & =-\frac{g_{x p} \bar{\lambda}}{\Omega} \mathcal{H}_{y y} & \frac{\partial y^{*}}{\partial p} & =\frac{g_{x p} \bar{\lambda}}{\Omega} \mathcal{H}_{x y} \\
\frac{\partial x^{*}}{\partial Z} & =\frac{\mathcal{H}_{x y} \mathcal{H}_{y Z}-\mathcal{H}_{x Z} \mathcal{H}_{y y}}{\Omega} & \frac{\partial y^{*}}{\partial Z} & =\frac{\mathcal{H}_{x y} \mathcal{H}_{x Z}-\mathcal{H}_{y Z} \mathcal{H}_{x x}}{\Omega} \\
\frac{\partial x^{*}}{\partial A} & =\frac{\partial x^{*}}{\partial M}=0 & \frac{\partial y^{*}}{\partial A} & =\frac{\partial y^{*}}{\partial M}=0 \\
\frac{\partial x^{*}}{\partial \mu} & =\frac{f_{y} \mathcal{H}_{x y}-f_{x} \mathcal{H}_{y y}}{\Omega} & \frac{\partial y^{*}}{\partial \mu} & =\frac{f_{x} \mathcal{H}_{x y}-f_{y} \mathcal{H}_{x x}}{\Omega} \\
\frac{\partial x^{*}}{\partial \lambda} & =-\frac{\mathcal{H}_{x y}+g_{x} \mathcal{H}_{y y}}{\Omega} & \frac{\partial y^{*}}{\partial \lambda} & =\frac{\mathcal{H}_{x x}+g_{x} \mathcal{H}_{x y}}{\Omega}
\end{aligned}
$$

where

$$
\Omega=\mathcal{H}_{x x} \mathcal{H}_{y y}-\mathcal{H}_{x y}^{2}
$$

which is positive by strict concavity. Then consider equations (36), (37) and (38), which equal zero in steady state, and apply again Cramer's rule to obtain:

$$
\begin{aligned}
Z_{p}^{L}= & \frac{\rho \bar{\lambda}}{\Omega|J|}\left[g_{x p}\left(f_{Z}-\rho\right)\left(f_{x} \mathcal{H}_{y y}-f_{y} \mathcal{H}_{x y}\right)\right. \\
& \left.+f_{x}^{2} g_{p Z} \mathcal{H}_{y y}+f_{y}^{2}\left(g_{p Z} \mathcal{H}_{x x}-g_{x p} \mathcal{H}_{x Z}\right)+\left(g_{x p} \mathcal{H}_{y Z}-2 g_{p Z} \mathcal{H}_{x y}\right) f_{x} f_{y}\right] \\
A_{p}^{L}= & \frac{\bar{\lambda}}{\Omega|J|}\left\{\left[\left(\mathcal{H}_{y Z}+g_{Z} \mathcal{H}_{y y}\right) f_{x}-\left(\mathcal{H}_{x y}+g_{x} \mathcal{H}_{y y}\right) f_{Z}\right]\left[\left(f_{Z}-\rho\right) g_{x p}-f_{x} g_{p Z}\right]\right. \\
& +\left[g_{x}\left(g_{x p} \mathcal{H}_{Z Z}-g_{p Z} \mathcal{H}_{x Z}\right)+g_{p Z}\left(g_{Z} \mathcal{H}_{x x}-g_{x} \mathcal{H}_{x Z}\right)+g_{Z}\left(g_{x}-g_{x p}\right) \mathcal{H}_{x Z}\right] f_{y}^{2} \\
& -\left[g_{x p}\left(\mathcal{H}_{Z Z}+g_{Z} \mathcal{H}_{y Z}\right)-g_{p Z}\left(\mathcal{H}_{x Z}+2 g_{Z} \mathcal{H}_{x y}-g_{x} \mathcal{H}_{y Z}\right)\right] f_{x} f_{y} \\
& -\left[\left(\mathcal{H}_{x x}+g_{x} \mathcal{H}_{x y}\right) g_{p Z}-g_{x p}\left(\mathcal{H}_{x Z}+g_{x} \mathcal{H}_{y Z}\right)\right] f_{Z} f_{y} \\
& \left.+\left(g_{x} \mathcal{H}_{y Z}-g_{Z} \mathcal{H}_{x y}\right)\left(f_{Z}-\rho\right) f_{y} g_{x p}\right\}+g_{p} A_{M}^{L} \\
\mu_{p}^{L}= & \frac{\rho \bar{\lambda}}{\Omega|J|}\left[\left(g_{x p} \mathcal{H}_{y Z}-g_{p Z} \mathcal{H}_{x y}\right)\left(f_{Z} \mathcal{H}_{x y}-f_{x} \mathcal{H}_{y Z}\right)-\left(g_{x p} \mathcal{H}_{Z Z}-g_{p Z} \mathcal{H}_{x Z}\right)\left(f_{y} \mathcal{H}_{x y}-f_{x} \mathcal{H}_{y y}\right)\right. \\
& \left.+\left(g_{x p} \mathcal{H}_{x Z}-g_{p Z} \mathcal{H}_{x x}\right)\left(f_{y} \mathcal{H}_{y Z}-f_{Z} \mathcal{H}_{y y}\right)\right] .
\end{aligned}
$$

Expressions (55) and (56) describe the long-run price effect on $Z$ and $A$. The long-run price responses for $x$ and $y$ are found by replacing the above expressions in (47) and (48), which for the general case yields

$$
\begin{aligned}
x_{p}^{L} & =\theta\left[f_{x} g_{p Z}-\left(f_{Z}-\rho\right) g_{x p}\right] f_{Z} \mathcal{H}_{y y}+D f_{y} \\
y_{p}^{L} & =\theta\left[f_{x} g_{p Z}-\left(f_{Z}-\rho\right) g_{x p}\right]\left(f_{x} \mathcal{H}_{y Z}-f_{Z} \mathcal{H}_{x y}\right)+E f_{y}
\end{aligned}
$$


where

$$
\begin{aligned}
\theta & =-\frac{\bar{\lambda} \rho}{\Omega|J|} \\
D & =\theta\left\{g_{p Z}\left(f_{y} \mathcal{H}_{x Z}-f_{Z} \mathcal{H}_{x y}-f_{x} \mathcal{H}_{y Z}\right)+g_{x p}\left[\mathcal{H}_{y Z}\left(2 f_{Z}-\rho\right)-f_{y} \mathcal{H}_{Z Z}\right]\right\} \\
E & =\theta\left[g_{p Z}\left(f_{Z} \mathcal{H}_{x x}-f_{x} \mathcal{H}_{x Z}\right)+g_{x p}\left(f_{x} \mathcal{H}_{Z Z}-f_{Z} \mathcal{H}_{x Z}\right)\right] .
\end{aligned}
$$

These general expressions are not particularly insightful per se. Notably, their formulation simplifies considerably in specific economic applications of interest.

For instance, when $f_{y}=0$, as in the class of models considered in Section 2, the long-run response of $x$ and $Z$ boil down to equations (10) and (11) in Proposition 1,

$$
\begin{aligned}
x_{p}^{L} & =a\left[f_{x} g_{p Z}-\left(f_{Z}-\rho\right) g_{x p}\right] f_{Z} \\
Z_{p}^{L} & =-a\left[f_{x} g_{p Z}-\left(f_{Z}-\rho\right) g_{x p}\right] f_{x},
\end{aligned}
$$

where

$$
a=\theta \mathcal{H}_{y y}=-\frac{\bar{\lambda} \rho}{\Omega|J|} \mathcal{H}_{y y}<0 .
$$

Moreover, in a consumer problem $g_{p Z}=0$ and $g_{x p}=-1$, hence (63) and (64) further simplify to equations (15) and (16):

$$
\begin{aligned}
x_{p}^{L} & =a\left(f_{Z}-\rho\right) f_{Z} \\
Z_{p}^{L} & =-a\left(f_{Z}-\rho\right) f_{x} .
\end{aligned}
$$

As a side result, note that the same procedure can be used to compute the response to a change in the exogenous component of income. Consider first the long run response:

$$
\begin{aligned}
x_{M}^{L} & =\frac{\partial x^{*}}{\partial M}+\frac{\partial x^{*}}{\partial Z} Z_{M}^{L}+\frac{\partial x^{*}}{\partial A} A_{M}^{L}+\frac{\partial x^{*}}{\partial \lambda} \frac{\partial \bar{\lambda}}{\partial M}+\frac{\partial x^{*}}{\partial \mu} \mu_{M}^{L} \\
y_{M}^{L} & =\frac{\partial y^{*}}{\partial M}+\frac{\partial y^{*}}{\partial Z} Z_{M}^{L}+\frac{\partial y^{*}}{\partial A} A_{M}^{L}+\frac{\partial y^{*}}{\partial \lambda} \frac{\partial \bar{\lambda}}{\partial M}+\frac{\partial y^{*}}{\partial \mu} \mu_{M}^{L} .
\end{aligned}
$$

Since $\partial x^{*} / \partial A=\partial x^{*} / \partial M=\partial y^{*} / \partial A=\partial y^{*} / \partial M=\partial Z^{L} / \partial M=\partial \bar{\lambda} / \partial M=\partial \mu^{L} / \partial M=0$, we obtain that, when the marginal utility of wealth is maintained constant, the long run dynamic income effects at the steady state are nil.

\section{A.3 Short-run substitution effects}

To compute short-run price effects, consider (34) and (35) and recall that in the short run state variables $A$ and $Z$ are given. Given that $\lambda$ is fixed, the short-run responses of $x$ and $y$, 
when all variables are at their steady-state level, only depend on the direct effect of price, and on its indirect effect through the costate variable $\mu$ :

$$
\begin{aligned}
& x_{p}^{S}=\frac{\partial x^{*}}{\partial p}+\frac{\partial x^{*}}{\partial \mu} \mu_{p}^{S} \\
& y_{p}^{S}=\frac{\partial y^{*}}{\partial p}+\frac{\partial y^{*}}{\partial \mu} \mu_{p}^{S} .
\end{aligned}
$$

The terms $\partial x^{*} / \partial p, \partial x^{*} / \partial \mu, \partial y^{*} / \partial p, \partial y^{*} / \partial \mu$ are described in equations (49) and (52). To obtain $\mu_{p}^{S}$, we need to assess how the saddle path of $\mu$ responds to a price change. Even without knowing its specific functional form, we can approximate it around the steady state. Take a first-order linear expansion of (36), (37) and (38):

$$
\left(\begin{array}{c}
\dot{Z} \\
\dot{A} \\
\dot{\mu}
\end{array}\right)=J \cdot\left(\begin{array}{c}
Z-Z^{L} \\
A-A^{L} \\
\mu-\mu^{L}
\end{array}\right)
$$

Consider the eigenvector $\left(1, \xi_{2}, \xi_{3}\right)$ associated to the negative eigenvalue $\varepsilon$ of the Jacobian matrix $J$, with

$$
\begin{aligned}
\xi_{3} & =\frac{1}{\Phi}\left[\left(\varepsilon-f_{Z}\right) \Omega+f_{x}\left(\mathcal{H}_{x Z} \mathcal{H}_{y y}-\mathcal{H}_{x y} \mathcal{H}_{y Z}\right)+f_{y}\left(\mathcal{H}_{x x} \mathcal{H}_{y Z}-\mathcal{H}_{x Z} \mathcal{H}_{x y}\right)\right] \\
\varepsilon & =\frac{1}{2}\left(\rho-\sqrt{\rho^{2}-4 \frac{|J|}{\rho}}\right)<0 \\
\Phi & =-f_{y}^{2} \mathcal{H}_{x x}+2 f_{x} f_{y} \mathcal{H}_{x y}-f_{x}^{2} \mathcal{H}_{y y}>0 .
\end{aligned}
$$

The particular solution of the system of ordinary linear differential equations (72) is

$$
\begin{aligned}
& Z(t)=Z^{L}+\alpha e^{\varepsilon t} \\
& A(t)=A^{L}+\alpha e^{\varepsilon t} \xi_{2} \\
& \mu(t)=\mu^{L}+\alpha e^{\varepsilon t} \xi_{3}
\end{aligned}
$$

where $\alpha$ is a constant that depends on initial values. Replacing $\alpha e^{\varepsilon t}=Z(t)-Z^{L}$ in (78), yields the feedback solution of $\mu$ as a function of the state variable $Z$ only,

$$
\hat{\mu}(Z)=\mu^{L}+\left(Z-Z^{L}\right) \xi_{3} .
$$

Taking the derivative of (79) with respect to $p$ yields, for any $A$, the short-run effect of a price change is

$$
\mu_{p}^{S} \equiv \frac{\partial \hat{\mu}(Z)}{\partial p}=\mu_{p}^{L}-\xi_{3} Z_{p}^{L}+\left(Z-Z^{L}\right) \frac{\partial \xi_{3}}{\partial p}
$$


In proximity of $Z^{L}$, the last term is negligible and the above expression simplifies to

$$
\mu_{p}^{S}=\mu_{p}^{L}-\xi_{3} Z_{p}^{L}
$$

Replacing (81) in (70)-(71) and rearranging yields the short-run price response of $x$ and $y$ :

$$
\begin{aligned}
x_{p}^{S} & =C\left(f_{x} \mathcal{H}_{y y}-f_{y} \mathcal{H}_{x y}\right)+\frac{\bar{\lambda}}{\Phi} f_{y}^{2} g_{x p} \\
y_{p}^{S} & =C\left(f_{y} \mathcal{H}_{x x}-f_{x} \mathcal{H}_{x y}\right)-\frac{\bar{\lambda}}{\Phi} f_{x} f_{y} g_{x p}
\end{aligned}
$$

where

$$
C=\varepsilon \theta\left\{g_{p Z}+\frac{g_{x p}}{\Phi}\left[\left(f_{x} \mathcal{H}_{y y}-f_{y} \mathcal{H}_{x y}\right)\left(f_{Z}-\rho\right)+\left(f_{y} \mathcal{H}_{x Z}-f_{x} \mathcal{H}_{y Z}\right) f_{y}\right]\right\}
$$

When $f_{y}=0$ the above expressions considerably simplify. For example, the short-run response of $x$ becomes

$$
x_{p}^{S}=a \varepsilon\left[f_{x} g_{p Z}-\left(f_{Z}-\rho\right) g_{x p}\right],
$$

as shown in equation (9) of Proposition 1.

In a consumer problem $g_{p Z}=0$ and $g_{x p}=-1$, hence (85) further simplifies to

$$
x_{p}^{S}=a \varepsilon\left(f_{Z}-\rho\right)
$$

as shown in equation (14).

Note that neither expression depends on assets or income, because income effects are nil also in the short run. To see it, consider

$$
\begin{aligned}
x_{M}^{S} & =\frac{\partial x^{*}}{\partial M}+\frac{\partial x^{*}}{\partial \mu} \mu_{M}^{S} \\
y_{M}^{S} & =\frac{\partial y^{*}}{\partial M}+\frac{\partial y^{*}}{\partial \mu} \mu_{M}^{S}
\end{aligned}
$$

Taking the derivative of (79) with respect to $M$ yields

$$
\mu_{M}^{S}=\mu_{M}^{L}-\xi_{3} Z_{M}^{L}=0
$$

Together with the fact that $\partial x^{*} / \partial M=\partial y^{*} / \partial M=0$, we conclude that $x_{M}^{S}=y_{M}^{S}=0$. 


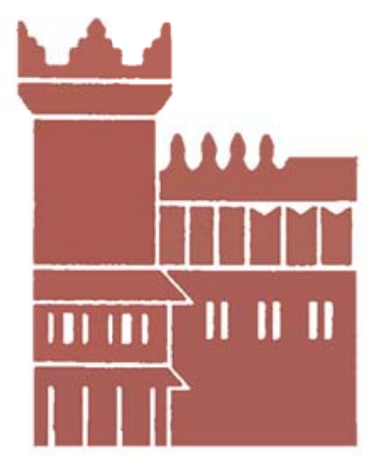

Alma Mater Studiorum - Università di Bologna DEPARTMENT OF ECONOMICS

Strada Maggiore 45

40125 Bologna - Italy

Tel. +39051 2092604

Fax +390512092664

http://www.dse.unibo.it 